\title{
SEGURANCA DO PACIENTE: PERCEPCÕES DE DOCENTES E DISCENTES DO CURSO TÉCNICO EM ENFERMAGEM
}

\author{
Cláudia Rodrigues ${ }^{1}$, Pétala Salvador ${ }^{1}$, Kisna Alves $^{1}$, Amanda Lopes ${ }^{2}$ \\ e Clara Marques ${ }^{3}$ \\ ${ }^{1}$ Escola de Saúde da Universidade Federal do Rio Grande do Norte (ESUFRN), Natal-RN, Brasil, \\ claudiacrisfm@yahoo.com.br; petatlatuani@hotmail.com \\ ${ }^{2}$ Gestora Hospitalar em enfermagem, Natal-RN, Brasil, amandalm_90@hotmail.com \\ ${ }^{3}$ Universidade Federal do Rio Grande do Norte (UFRN), Natal-RN, Brasil, claraluciacs@gmail.com
}

\begin{abstract}
Resumo. Introdução: A segurança do paciente é um dos assuntos relevantes na área da saúde. Justifica-se a importância de estudar essa temática como forma de identificar o que ela representa para professores e alunos da área da enfermagem. Objetivos: Investigar as percepções de docentes e discentes de enfermagem acerca da segurança do paciente. Métodos: Estudo descritivo, transversal, de abordagem qualitativa. Os dados textuais provenientes das entrevistas com docentes do curso técnico em enfermagem e de um grupo focal realizado com os estudantes do último ano do referido curso. Esses dados foram transcritos e analisados com suporte do software Interface de $R$ pour Analyses Multidimensionnelles de Textes et de Questionneires (IRAMUTEQ®). Resultados: Com a analise dos dados emergiram cinco classes de conteúdo: 1) a educação permanente como necessária à segurança do paciente $(18,9 \%) ; 2$ ) assistência direcionada para a segurança $(17,6 \%)$; 3) risco de erro no âmbito de saúde $(13,1 \%)$; 4) conduta para a assistência segura $(26,8 \%) ; 5)$ procedimentos de enfermagem associados à segurança $(23,5 \%)$. Conclusões: Os entrevistados compreenderam que a segurança do paciente está baseada em três características primordiais: assistência de qualidade, técnica, conhecimento.
\end{abstract}

Palavras-chave: Segurança do Paciente; Enfermagem; Educação Permanente; Qualidade da Assistência à Saúde.

\section{PATIENT SAFETY: PERCEPTIONS OF TEACHERS AND STUDENTS OF THE TECHNICAL NURSING COURSE}

\begin{abstract}
Introduction: Patient safety is one of the relevant issues in the health area. The importance of studying this topic is justified as a way of identifying what it represents for teachers and students in the field of nursing. Objectives: To investigate the perceptions of nursing professors and students about patient safety. Methods: Descriptive, cross-sectional study with a qualitative approach. The textual data from the interviews with professors of the technical nursing course and from a focus group conducted with the students of the last year of that course. These data were transcribed and analysed using the software Interface de R pour Multidimensionnelles de Textes et de Questionneires (IRAMUTEQ®). Results: With the analysis of the data, five content classes emerged: 1) permanent education as necessary for patient safety $(18.9 \%)$; 2) assistance directed towards security $(17.6 \%)$; 3 ) risk of error in the health field $(13.1 \%) ; 4)$ conduct for safe assistance $(26.8 \%)$; 5) nursing procedures associated with safety $(23.5 \%)$. Conclusions: Respondents understood that patient safety is based on three primary characteristics: quality care, technique, knowledge.
\end{abstract}

Keywords: Patient safety; Nursing; Permanent Education; Quality of Health Care.

\section{INTRODUÇÃO}

A segurança do paciente é considerada pelas instituições de saúde como uma das metas a serem alcançadas afim de garantir uma assistência livre de erros. Desse modo, a 
importância de boas práticas em saúde a partir de referências significativas e de teorias do cuidado em saúde são primordiais para a realização de ações capazes de contribuir para a prevenção de possíveis incidentes em saúde. A partir de 2004 a Organização Mundial da Saúde (OMS) lançou a Aliança Mundial para a Segurança do Paciente a fim de estabelecer nos seus países membros o compromisso em desenvolver políticas públicas que fortaleçam as práticas voltadas para a assistência segura (Cavalcante, 2015). No Brasil, em 2013, foi elaborado o Programa Nacional de Segurança do Paciente (PNSP), com o intuito de contribuir para a qualificação do cuidado em todos os estabelecimentos de saúde, bem como possibilitar o envolvimento da temática para os pacientes e familiares nas ações de segurança do paciente. Além disso, propõe ampliar o acesso da sociedade às informações relativas ao tema a fim de sistematizar conhecimentos sobre a segurança do paciente e fomentar a inclusão do tema no ensino técnico, de graduação e pós-graduação na área da saúde (Brasil, 2014).

Diante disso, a literatura destaca que há um desconhecimento dos profissionais de saúde acerca do cuidado seguro e este ainda é considerado como um grande empecilho para a promoção da segurança do paciente. Pois, essa temática ainda é vista com uma cultura de culpa e punições individuais por todos que compõe o trabalho em saúde (Cavalcante, 2015).

A equipe de enfermagem, em especial, são considerados elementos chaves para o fomento de ações que possa contribuir para a redução desses riscos. Afinal, são esses profissionais que possuiem um contato mais próximo com o paciente, estabelecendo um cuidado direto e realizando procedimentos de média e alta complexidade. E, por causa disso, membros primordiais no cuidado em saúde.

Entretanto, a formação desses profissionais ainda carece de instrumentalização para lidar com os riscos e as possíveis falhas que possam desenvolver no ambiente de trabalho em saúde. O fato de não saber lidar com o possíveis incidente no ambiente de saúde faz com que esses profissionais não reconheça possíveis falhas no decorrer da prestação dos cuidados.

Com isso, é indispensável para a qualidade do cuidado e adesão à prática da cultura de segurança do paciente de forma efetiva, torna-se relevante o envolvimento da gestão no processo assistencial.

Neste ínterim, a formação desse profissional deve englobar uma estrutura curricular em que a temática de segurança do paciente seja transversal e capaz de envolver docentes e 
discentes em medidas preventivas de danos nos variados cenários da assistência (Duarte, Stipp, Silva, \& Oliveira, 2015).

Nessa perspectiva, se faz necessário uma investigação de como professores estão promovendo o ensino acerca da segurança do paciente para esses futuros profissionais. Bem como, esses discentes compreendem essa temática.

Assim, este estudo apresenta uma investigação acerca do ensino da segurança do paciente no ensino técnico em enfermagem em uma Universidade Pública do Brasil. Partiu-se da seguinte questão norteadora: quais as percepções de segurança do paciente que os docentes e discentes do curso técnico em enfermagem possuem?

Desse modo, o presente estudo tem por objetivo investigar as percepções de docentes e discentes do curso técnico em enfermagem sobre a segurança do paciente.

\section{METODOLOGIA}

Trata-se de um estudo descritivo, transversal, de abordagem qualitativa. Realizado em uma Universidade Federal do Nordeste do Brasil. Além de outros cursos, a referida universidade oferta o curso técnico de enfermagem há 65 anos, sendo uma instituição consolidada na trajetória de enfermagem no nordeste (ESUFRN, 2018).

Desse modo, elegeu-se tal instituição de ensino afim de conhecer de que forma se dá a percepção dos docentes e discentes acerca da temática segurança do paciente. Assim, a pesquisa teve a sua coleta de dados dividida em dois momentos. No primeiro deles foi realizada uma entrevista semi-estrutudada com os professores que compõe o corpo docente da instituição.

Para participar do estudo elegeu-se como critérios de inclusão: ser docentes efetivos que atuavam em unidade curriculares que integram o curso técnico em enfermagem e aceitar, voluntariamente, a participar da pesquisa.

Excluiu-se os docentes em afastamento, férias ou atestado de qualquer natureza, bem como os docentes responsáveis pela coordenação da referida pesquisa.

Neste ínterim, obedecendo os critérios de inclusão acima citados a amostra final foi composta por 21 docentes. Houve 1 recusa e 5 professores estavam em afastamento no período da coleta de dados. 
Esse momento da coleta de dados ocorreu entre os meses de setembro de 2018 a março de 2019. As entrevistas foram do tipo semi-estruturada individual e com gravadas um auxílio de um gravador digital. Os dados foram coletados no próprio ambiente de trabalho, por meio de agendamento prévio em horários definidos pelos participantes.

No segundo momento da coleta de dados, foi realizado um grupo focal com os discentes do curso técnico em enfermagem matriculados regularmente no último período do curso, elegeu-se o ultimo período do curso por compreender que os discentes já tiveram contato com a temática segurança do paciente em momentos teóricos e práticos.

Assim, elegeu-se como critérios de exclusão: o discente não estar regular nos módulos e componentes curriculares, estar cursando outro período do curso.

Então, a população dos discentes constituiu-se de 29 alunos que estavam regularmente matriculados.

A coleta de dados se deu através da técnica de grupo focal, essa caracteriza-se como uma estratégia para avaliação e obter de dados por meio das informações qualitativas sendo essencial para o objeto de pesquisa. Nela os moderadoes iniciam com perguntas amplas e que estimulam a interação dos participantes no momento da coleta de dados, o que passa a explorar os pontos de vista e questões relacionadas a temática. (Tong, Sainsbury, \& Craig, 2007).

Assim, foi agendado previamente dia e horário para a realização do grupo focal, em que compareceram 10 alunos, estes compuseram a amostra final do estudo. O grupo focal foi realizado no laboratório de práticas de enfermagem da referida escola. O ambiente estava previamente preparado, e contou com a participação de uma facilitadora e duas relatoras que auxiliaram na execução da atividade, além dos 10 discentes entrevistados.

Durante o grupo, foram esclarecidas as dúvidas referentes a proposta de coleta de dados e sobre a temática, entregue os Termos de Consentimento Livre e Esclarecimento (TCLE) e Autorização para Gravação de Voz, sendo iniciada após as assinaturas dos termos de todos os participantes. Ao final da discussão grupo teve a duração de 38 minutos.

Aos docentes e os discentes convidados para a participação da pesquisa, foram utilizados dois instrumentos de pesquisa, composto por: 1) questionário de caracterização, de autopreenchimento pelo sujeito de pesquisa; e 2) Roteiro de realização da entrevista, 
composto por duas questões: a) O que você compreende por Segurança do Paciente? b) quais ações você associa a esta temática?

Para os docentes, o questionário de caracterização foi composto por perguntas sobre idade, sexo, maior titulação acadêmica e experiência curricular acerca da segurança do paciente. Enquanto aos discentes foi questionado idade, sexo, curso e se já atuou como profissional em algum serviço de saúde.

Os dados textuais provenientes das entrevistas com os docentes e discentes foram transcritos e analisados com suporte do software Interface de $R$ pour Analyses Multidimensionnelles de Textes et de Questionneires (IRAMUTEQ ${ }^{\circledR}$ ), o qual processa análises lexicais de dados textuais ao fornecer contextos e classes por meio do julgamento da semelhança de seus vocabulários, de maneira a contribuir na compreensão do ambiente de sentido das palavras, logo, indicar elementos representativos do objeto estudado (Camargo \& Justo, 2013).

Para esta finalidade, utilizou-se a Classificação Hierárquica Descendente (CHD) e a análise de similitude como método de tratamento dos dados, o que possibilitou a identificação da frequência de cada palavra e sua conexão com as outras, além de auxiliar na análise do corpus textual (Camargo \& Justo, 2013).

A análise do corpus proveniente da transcrição das 31 entrevistas semiestruturadas resultou em 6990 ocorrências de palavras, apresentadas em 1416 formas distintas, como ponte de corte para a análise de similitude seguiu-se o cálculo: duas vezes a frequência média de ocorrências para cada forma.

Ressalta-se que a interpretação e análise dos dados teve embasamento da literatura atual no que concerne à segurança do paciente e à gestão da qualidade em saúde. O estudo seguiu os princípios éticos e legais que regem a pesquisa científica em seres humanos, preconizados na Resolução $n^{\circ} 466 / 2012$, do Conselho Nacional de Saúde. Sendo submetido ao comitê de ética da referida universidade e aprovado conforme protocolo CAAE n`88024618.3.0000.5537. 


\section{RESULTADOS}

A caracterização dos docentes que participaram da pesquisa, $19(90,5 \%)$ eram do sexo feminino e $3(9,5 \%)$ do sexo masculino, com idades entre 30 a 63 anos, 17 (81\%) possuíam o título de doutor e os demais $4(19 \%)$ mestres.

Já entre os discentes que participaram do grupo focal, predominou a idade de 19 a 34 anos, sendo $8(80 \%)$ sexo feminino e $2(20 \%)$ do sexo masculino, 5 (50\%) dos discentes possuíam formação acadêmica anterior.

Com relação ao corpus textual podemos dizer que a partir da análise dos dados com 0 auxílio do Iramuteq ${ }^{\circledR}$ resultou em 5 classes, a saber: 1) a educação permanente como necessária à segurança do paciente $(18,9 \%) ; 2)$ assistência direcionada para a segurança $(17,6 \%)$; 3) risco de erro no âmbito de saúde(13,1\%); 4) conduta para a assistência segura $(26,8 \%) ; 5)$ procedimentos de enfermagem associados à segurança (23,5\%). Como demonstrado na Figura 1, a seguir:

\begin{tabular}{|c|c|c|c|c|c|c|c|c|c|c|c|c|c|c|}
\hline & & & & & \multicolumn{5}{|c|}{$\begin{array}{l}\text { Percepções de docentes e discentes acerca da } \\
\text { compreensão da segurança do paciente }\end{array}$} & & & & & \\
\hline \multirow{2}{*}{\multicolumn{3}{|c|}{$\begin{array}{c}\text { Classel (18,9) } \\
\text { A educcaçăopermanente comonecesśriaà } \\
\text { segurança do paciente }\end{array}$}} & \multicolumn{3}{|c|}{$\begin{array}{c}\text { Classel|(17,6) } \\
\text { Assistência direcionada para a segurança }\end{array}$} & \multicolumn{3}{|c|}{$\begin{array}{c}\text { Classelll(13,1\%) } \\
\text { Riscodeerro noâmbitode saúde }\end{array}$} & \multicolumn{3}{|c|}{$\begin{array}{c}\text { Classe IV (26,8\%) } \\
\text { Conduta para assisêtencia segura }\end{array}$} & \multirow{2}{*}{\multicolumn{3}{|c|}{$\begin{array}{c}\text { ClasseV }(23,5) \\
\text { Procedimentos de enfermagem associados à } \\
\text { segurança }\end{array}$}} \\
\hline & & & \multirow{2}{*}{ Palavra } & \multirow[t]{2}{*}{$\%$} & \multirow{2}{*}{$\begin{array}{c}\text { Variável } \\
\text { significativa }\end{array}$} & Palavra & \multirow[t]{2}{*}{$\%$} & \multirow{2}{*}{$\begin{array}{c}\text { Variâvel } \\
\text { significativa }\end{array}$} & \multirow[t]{2}{*}{ Palavra } & \multirow[t]{2}{*}{$\%$} & \multirow{2}{*}{$\begin{array}{l}\text { Variável } \\
\text { significativa } \\
<0,0001\end{array}$} & & & \\
\hline Palavra & $\%$ & $\begin{array}{l}\text { Variável } \\
\text { significativa }\end{array}$ & & & & & & & & & & Palavra & $\%$ & $\begin{array}{l}\text { Variável } \\
\text { significativa }\end{array}$ \\
\hline Educação & $100 \%$ & $<0,0001$ & Técnicas & $85,71 \%$ & $<0,0001$ & Proceament & & 0001 & Qualidade & $75,0 \%$ & $<0,0274$ & Identificaậa & $100 \%$ & $<0,0001$ \\
\hline Profisssional & $32,14 \%$ & $<0,0488$ & Correto & $75,0 \%$ & $<0,0001$ & Medicação & $50,0 \%$ & $<0,0003$ & Protocolo & $75,0 \%$ & $<0,0274$ & Registro & $100 \%$ & $<0,0002$ \\
\hline Paciente & $28,78 \%$ & $<0,0086$ & Administrar & $66,0 \%$ & $<0,0115$ & Errar & $66,67 \%$ & $<0,0054$ & & & . & Lesão & $100 \%$ & $<0,0016$ \\
\hline Situação & $100 \%$ & $<0,0029$ & Utilização & $66,67 \%$ & $<0,0244$ & Hospital & $50,0 \%$ & $<0,0014$ & Risco & $63,16 \%$ & $<0,0001$ & Comunicação & $71,43 \%$ & $<0,0022$ \\
\hline Entender & $75,0 \%$ & $<0,0001$ & Segurança & $67,0 \%$ & $<0,0001$ & Possivel & $100 \%$ & $<0,0001$ & Proteção & $83,33 \%$ & $<0,0014$ & Queda & $83,33 \%$ & $<0,0004$ \\
\hline
\end{tabular}

Figura 1. Classes geradas a partir da classificação hierárquica descendente das percepções de docentes e discentes acerca da compreensão e das ações de enfermagem voltadas para a segurança do paciente.

Na classe I "A educação permanente necessária a Segurança do Paciente" foi possível identificar que os discentes e docentes consideram a segurança do paciente como um cuidado básico o qual se reflete na qualidade de assistência, sendo necessário que o profissional busque aprimorar suas habilidades através da adesão à estratégias educativas, como pode ser visto nas falas abaixo:

“(...) O profissional deve estar realmente capacitado para poder colocar em prática as ações correspondentes os diversos tipos de patologia dependendo do quadro clínico daquele paciente, ressalto muito isso a questão do saber." (Discente 10) 
Na classe II "Assistência direcionada para a segurança" identificou-se a importância do profissional técnico em enfermagem em reconhecer as ações que reforcem a prática da segurança do paciente, sendo considerada pelos docentes e discentes que as técnicas corretas e a utilização dos equipamentos de proteção individual, são fundamentais para a assistência à saúde com qualidade, como pode ser visto nas falas abaixo:

“Os procedimentos são realizados na administração de medicamentos, no cuidado com o curativo do paciente na prevenção de contaminação de algum material estéril para realizar um procedimento, a técnica correta para administrar o medicamento." (Discente 09)

$\mathrm{Na}$ classe III "Procedimentos que possuem risco de falha no Âmbito de Saúde" foi visto pelos docentes os principais riscos atribuídos à assistência de enfermagem, bem como as causas que afetam o gerenciamento do cuidado nos serviços de saúde, como pode ser visto nas falas abaixo:

“É importante ter muita atenção, porque a segurança na verdade é tudo, aborda não somente os procedimentos invasivos, mais também o cuidado com a cirurgia, a esterilização, bem como os procedimentos que impera maiores riscos e que devemos ter muito cuidado." (Docente 08)

Com relação a classe IV "Conduta necessária para a qualidade da Assistência Segura", os docentes e discentes consideraram de suma importância para a redução dos riscos e danos ao paciente o conhecimento técnico/científico dos profissionais, bem como o acesso aos protocolos acerca da segurança do paciente com o intuito de promover uma assistência à saúde segura e eficiente, como pode ser visto nas falas abaixo:

\footnotetext{
"A segurança do paciente está ligada aos protocolos de segurança e prevenção que a gente tem sobre a segurança do paciente associado a área da higiene das mãos primordialmente a uma assistência limpa, segura e prioritária.” (Discente 07)
}

Na classe V "Ações e Procedimentos de Enfermagem associados à Segurança do Paciente" remete as ações de cuidados que envolve a temática segurança do paciente, sendo abordados pelos docentes como estratégias que permitem a atenção e o cuidado seguro e que favoreça a adoção de medidas preventivas e o monitoramento das atividades visando reduzir os riscos e danos aos pacientes associados através da prática assistencial inadequada durante sua estadia nos serviços de saúde, como pode ser visto nas falas abaixo: 
“As ações de relação com a prevenção de infecção, a prevenção de quedas, a prevenção de iatrogenias de erro ao administrar um medicamento. Existe uma gama de ações que estão relacionadas a esta temática de segurança do paciente." (Docente 01)

A Figura 2 representa a análise de similitude do corpus transcrito das entrevistas com os docentes. Na parte superior destaca-se a expressão segurança do paciente que remete as concepções dos docentes sobre a temática, enquanto a palavra paciente remete as vivências e dão origem as demais categorias.

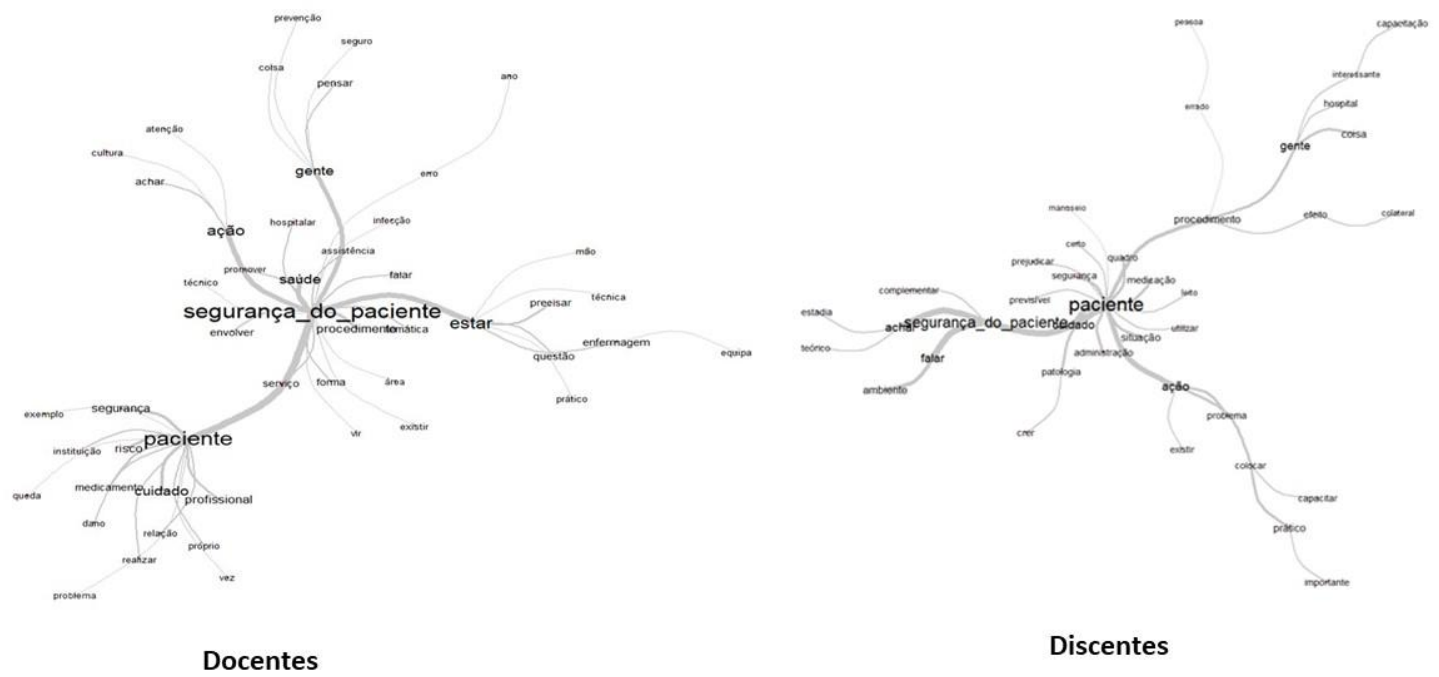

Figura 2. Análise de similitude das percepções e vivências docentes e discentes sobre a segurança do paciente.

Destaca-se que para os docentes as percepções que envolve o termo segurança do paciente estão descritas de forma a promover uma assistência segura e que envolve uma questão técnica, cultural e de prevenção. No que se refere as vivências, representado pela palavra paciente, destacam-se os procedimentos e cuidado direcionado. A Figura 2 representa a análise de similitude do corpus transcrito das entrevistas com os discentes. É importante perceber que o termo paciente encontra-se relacionado a vivência dos participantes, enquanto o termo segurança do paciente relaciona-se com a concepção.

Cabe ressaltar, que apesar da análise de similitude apresentar os mesmos termos em destaque os conceitos relacionados as percepções e vivencias são diferenciados. Enquanto, os docentes avaliam a segurança do paciente de forma a capacitar, envolver e de forma precisa os discentes avaliam de forma complementar aos procedimentos realizados. Já no 
que se refere as vivências, ambos tiveram aspectos semelhantes, de que os procedimentos devem estar em primeiro lugar no que se refere a atenção voltada para a segurança do paciente.

Por conseguinte, podemos observar a partir da análise dos resultados, é visto que a percepção dos docentes e discentes é de que a segurança do paciente é uma temática que compreende estratégias com vistas a reduzir os eventos adversos além de evitar falhas ocasionadas através das práticas inseguras que colocam em risco a vida e a saúde dos pacientes.

\section{DISCUSSÃO}

Com relação a caracterização dos sujeitos do estudo, foi visto que a maioria dos docentes e estudantes são do sexo feminino. Fato, este, que reflete um padrão mundial da enfermagem. Uma vez que, por ser reconhecida como a profissão do cuidar há uma tendência de ter em seu perfil profissionais mulheres. Entretanto, observa-se que há um número crescente de homens ingressando na enfermagem, esta é uma realidade nacional conforme a pesquisa sobre o perfil da enfermagem no Brasil (Cofen, 2013).

Além disso, foi visto que os professores são adultos jovens em plena idade produtiva para a realização de suas atividades, isso demonstra que a força de trabalho da referida escola é prinmordialmente jovem (Giordano \& Feli, 2017).

Já em relação aos discentes foi percebido que essa amostra é bastante diversifica, pois possui alunos que acabaram de sair do ensino médio e ingressaram no curso profissionalizante, como também discentes adultos jovens. Como também foi visto que esses metade dos alunos que participaram desse estudo já possuiam uma formação universitária anterior, fato este que refletiu nas discussões ocorridas no grupo focal.

No que se refere as percepções desses sujeitos quando se fala sobre a temática de segurança do paciente três aspectos básicos refletiram nas falas: a assistência de qualidade como elemento fundamental para o cuidado; a técnica realizada embasada em conceitos teóricos consistentes e o conhecimento que permeia todo esse campo como um elemento essencial para a realização de um cuidado seguro.

Neste ínterim, com base nas falas dos sujeitos é imprescindível que o ensino esteja consolidado em ações voltadas para os princípios da segurança do paciente, afim de que os 
profissionais já sejam formados com os direcionamentos das boas práticas na assistência em saúde e implementações de aspectos teóricos que fundamentem a cultura voltada para a segurança do paciente.

Sobre a qualidade da assistência, é imprescindível que os técnicos de enfermagem, estejam comprometidos e instrumentalizados sobre o real sentido da segurança do paciente, e que busquem melhorias constantes em sua prática profissional, o que pode ser representado pelo incentivo a educação permanente desses profissionais.

Nesta perspectiva, é de fundamental importância a participação efetiva do profissional técnico de enfermagem na garantia do cumprimento de normas e protocolos estabelecidos no ambiente de saúde acerca da segurança do paciente. Dentre as boas práticas podem ser citados, o cumprimento de ações através dos protocolos de segurança do paciente que estabelece base para as práticas de saúde e ações de gestão de risco, conforme as atividades desenvolvidas pelo serviço de saúde tendo como referencia os temas prioritários (Brasil, 2013).

Foi reconhecido ainda, pelos entrevistados que a assistência em saúde é por si só é um possível fator gerador de falhas. Afinal, podem afetar diretamente a saúde do paciente quando submetidas a cuidados inseguros. Sobre esse aspecto um estudo de Gomes et al. (2019) cita que eventos adversos podem acontecer e vão desde de uma comunicação ineficaz entre a equipe até a baixa continuidade da atenção prestada, o que pode gerar o comprometimento da assistência e com isso incidentes aos pacientes. Com isso, existem falhas referente a ambiência dos serviços de saúde ocasionadas pela grande demanda de pacientes na estrutura do serviço de saúde, assim como pela quantidade insuficiente de materiais e equipamentos para atender a necessidade do serviço, comprometendo também a qualidade da assistência e a segurança do paciente (Silva et al., 2016).

Diante do exposto, os discentes e os docentes desta pesquisa corroboram com a importância de que a falha na assistência pode ser vista como fonte de ensinamento e aprendizado para o trabalho em equipe, o que consolida assim, aspectos da cultura de segurança do paciente. Segundo Gomes (2019), a falha na assistência pode ser vista como uma oportunidade de adquirir uma visão holística do processo de trabalho e um espaço para mudanças que permitam contribuir para um modelo assistencial pautado na integralidade do paciente, com medidas de aprendizado contínuo e mudanças curriculares nos cursos de formação dos profissionais de saúde. 
Os entrevistados ainda destacaram, a necessidade de condutas que resultem numa assistência segura, como o uso de protocolos e checklist, uma vez que os profissionais são responsáveis pelo planejamento e intervenção apropriada com o objetivo de assegurar a redução do risco de danos desnecessários a saúde do paciente/cliente.

Segundo o estudo de Silva (2016) o cumprimento das ações da assistência à saúde reduziu significativamente o surgimento de doenças e agravos decorrentes do tempo de permanência dos pacientes submetidos ao cuidado, como também despesas desnecessárias ao hospital. Em contrapartida, verificou-se a importância da utilização de checklist, como uma medida simples, eficiente, eficaz e segura para a realização de procedimentos cirúrgicos proporcionando a equipe maior confiabilidade nas ações, pois permite a confirmação dos dados e informações necessárias do paciente (Silva et al., 2016).

Verifica-se, ainda, a importância dos profissionais da enfermagem nas ações de promoção, prevenção e no monitoramento com o objetivo de minimizar os riscos de danos aos pacientes associados ao cuidado em saúde. Por ser a categoria profissional com maior envolvimento na assistência do cuidado, faz-se necessário o comprometimento e adesão as boas práticas de segurança, bem como promover um atendimento humanizado assegurando a saúde do paciente (Teixeira et al., 2018).

Além disso, também foi relatado pelos participantes a importância da educação permanente na prática da segurança do paciente representada na classe I do corpus dos docentes e discentes, uma vez que o aprendizado possibilita uma reflexão crítica acerca das práticas de atenção à saúde, bem como proporciona aos profissionais a oportunidade de mudanças no processo de conhecimento no âmbito da segurança do paciente favorecendo um cuidado seguro.

\section{CONCLUSÕES}

Avaliou-se nesta pesquisa a percepção de docentes e discentes sobre a segurança do paciente. Foi visto que os sujeitos compreendem o real significado de promover uma cultura de segurança do paciente, sendo necessário que o profissional técnico de enfermagem além de possuir a habilidade técnica, precisa dispor do referencial técnico cientifico em benefício das boas práticas assistências visando a redução dos eventos adversos. 
Foi visto pelos docentes e discentes a importância de se instrumentalizar para melhor fazer o cuidado seguro na assistência, e que isso deve ser iniciado a partir da formação desse profissional. Ressalta, ainda, a relevância do técnico em enfermagem como um elemento fundamental no processo do cuidar, sendo essencial para a categoria aprimorar suas habilidades a fim de contribuir para uma assistência segura e de qualidade. Como limitação do estudo, destaca-se a dificuldade de marcação das entrevistas com os docentes, em virtude da carência de tempo hábil na agenda destes. Entretanto, ressalta-se que grande maioria aceitou participar voluntariamente da pesquisa.

Agradecimentos. Conselho Nacional de Pesquisa Científica - CNPq por subsidiar este estudo.

\section{REFERÊNCIAS}

Cavalcante, A. N., Rocha, R.C., Nogueira, L.T. Avelino, F.V.S.D., Rocha, S.S. (2015). Cuidado Seguro ao paciente: contribuições da enfermagem. Revista Cubana de Enfermería, 32(4), 1-13. Disponível em: http://www.revenfermeria.sld.cu/index.php/enf/article/view/907

Camargo, B. V. Justo, A. M. (2013). Iramuteq: um software gratuito para análise de dados textuais. Temas em psicologia, 21(2), 513-518. http://dx.doi.org/10.9788/TP2013.2-16

Conselho Federal de Enfermagem, Brasil. Perfil da enfermagem no Brasil (2013). Disponível em: http://www.cofen.gov.br/perfilenfermagem/blocoBr/QUADRO\%20RESUMO_Brasil_Final.pdf

Duarte, S. C. Stipp, M. A. C. Silva, M. M. Oliveira, F. T. (2015). Eventos adversos e a segurança do paciente na assistência em enfermagem. Revista Brasileira de enfermagem. 68(11), 1-11. https://doi.org/10.1590/0034$7167.2015680120 p$

Gomes, A. T. D. L., Ferreira Junior, M. A., Salvador, P. T. O. C., Bezerril, M. S., Chiavone F. B. T., Santos, V. E. P. (2019) Segurança do paciente em situação de emergência: percepções da equipe de enfermagem. Revista Brasileira de Enfermagem, 72(3), 752-759. https://doi.org/10.1590/0034-7167-2018-0544

Ministério da Saúde (2014). Documento de referência para o programa nacional de segurança do paciente. Fundação Oswaldo Cruz. Agência Nacional de Vigilância Sanitária.

Silva A., Carmelo, S. H. H., Terra F. S., Dázio, E. M. R., Sanches, R. S., Resk, Z. M. R. (2018) Segurança do paciente e a atuação do enfermeiro em hospital. Revista de Enfermagem UFPE-on-line. 12(6), 1-7. https://doi.org/10.5205/1981-8963-v12i6a234593p1532-1538-2018

Silva, A. T., Alves, M. G., Sanches, R. S., Terra, F. S., Resck, Z. M. R. (2016) Assistência de enfermagem e o enforque da segurança do paciente no cenário brasileiro. Saúde e debate, 40(111), 292-301. DOI: https://doi.org/10.1590/0103-1104201611123

Tong, A., Sainsbury, P., Craig, J. (2007) Consolidated criteria for reporting qualitative research (COREQ): a 32item checklist for interviews and focus groups. International Journal for Quality in Health Care; 19(6), 349 357. https://doi.org/10.1093/intqhc/mzm042

Wegner, W., Silva, S. C., Kantorski, K. J. C., Predbon, C. M., Sanches, M. O., Pedro, E. N. (2016) Educação para a cultura de segurança do paciente: implicações para a formação professional. Revista da Escola de Enfermagem Ana Nery. 20(3), 1-8. https://doi.org/10.5935/1414-8145.20160068 